\title{
COMPLEMENT FIXATION IN PULMONARY TUBERCULOSIS.
}

\author{
By J. A. D. RADCLIFFE, M.B., B.CH. \\ Pathologist, King Edward VII Sanatorium, Midhurst.
}

The application of the method of fixation of complement as a means of investigating immunity in tuberculosis dates from the experiment of Bordet and Gengou, in which they were able to demonstrate complement-fixing antibodies, against both avian and human tubercle bacilli, in the serum of guinea-pigs experimentally infected with avian bacilli. Gengou extended these experiments later, and showed that guinea-pigs infected with acid-fast bacilli, either pathogenic or saprophytic, developed complement-fixing antibodies not only against the homologous bacilli, but also against the other acid-fast bacteria. These observations of Gengou have been confirmed by other workers and consequently this reaction possesses only a relative specificity for tuberculosis, and must only be regarded as a group-reaction common to the acid-fast bacilli.

Wassermann and Bruck applied the method to the diagnosis of tuberculosis in man, using as antigen either old Tuberculin or the Tuberculin R. of Koch. They were, however, only able to demonstrate complement-fixing antibodies in the serum of patients who had been treated for a long time with tuberculin. Citron was the first to find these bodies in the serum of tuberculous persons who had not received any tuberculin. These results were confirmed by Lüdke, but were criticised by Morgenroth and Rabinowitsch who attributed them to "summation," and found that the serum of healthy persons might give similar results.

Numerous investigations have, since then, been carried out, both in cases of natural infection and in experimental tuberculosis, but the results have been very divergent, particularly among the earlier workers on the subject. The observers who have obtained the best 
results have only succeeded in getting a positive finding in about $50 \%$ of the cases, and generally only when the disease was in an advanced stage.

The results in bovine tuberculosis are no better, with the exception of those obtained by Hammer, who obtained $100 \%$ of positive results in the case of 48 tuberculous bovine sera, but among 48 normal animals two were positive. Hammer used as his antigen a mixture of bovine tuberculin and an acetone-alcohol extract of tuberculous tissue. His results have not been confirmed by other workers using the same technique, e.g., Bang and Andersen.

Among the more recent publications on the subject, those of Besredka and of Calmette are the most important and appear to be much more reliable than the earlier works. Besredka and his co-workers use a special egg-bouillon for the production of the antigen, and obtain about $90 \%$ of positive results in tuberculous cases. The value of their method is, however, impaired by the fact that syphilitic sera also give positive findings, so that the method cannot be regarded as a specific one. Renaux claims to have overcome this difficulty by extracting the Besredka antigen with sulphuric ether and so removing from it the fatty bodies. This treatment is said not to affect the value of the antigen in tuberculosis, whilst it no longer gives inhibition of haemolysis with syphilitic serum.

Calmette and Massol prepare their antigen by extracting washed tubercle bacilli with a $1 \%$ solution of Witte's peptone at a temperature of $65^{\circ}$, filtering and concentrating the filtrate. Using this antigen these authors have obtained $92.5 \%$ of positive results in 134 cases of human tuberculosis. I have not, however, seen any confirmation of their results by other workers, and a few experiments carried out here did not give very reliable findings, owing to the amount of "Eigenhemmung" in the antigen.

The great disparity in the results obtained by different observers is in all probability due mainly to differences in technique, and particularly to the use of one or other of the tuberculins as antigen. It is now recognised, however, that tuberculin is not a suitable antigen for this work, and that, in addition, it possesses such marked "Eigenhemmung" qualities that errors due to summation effects are very likely to be met with.

The use of alcoholic extracts either of tuberculous tissue or of tubercle bacilli is also unsatisfactory, as these extracts may act as syphilitic antigens and so give the Wassermann reaction. 
On account of these differences and uncertainties in results, it is impossible to compare the work of different observers, and consequently it is unnecessary to discuss the literature of the subject.

In this communication I have embodied (Part I) the results obtained when using an antigen which, whilst relatively easy to prepare, is less likely to give rise to errors due to unspecific absorption. In Parts II and III the effects of certain alterations in the technique are discussed.

\section{PART I.}

\section{Technique.}

The general arrangement of the test conforms to that of the original Wassermann reaction, and so does not require detailed description. It may be mentioned, however, that each substance used in the test (viz. serum, antigen, complement, haemolytic amboceptor and blood) was contained in a volume of $0.5 \mathrm{c.cm}$., so that the total volume in each tube at the end of the experiment was $2.5 \mathrm{c.cm}$.

Of the substances employed in the test, two, viz. antigen and complement, demand careful consideration, and will be dealt with in detail, the others are not subject to much variation and only require a few words of explanation.

(1) Serum. The serum for examination has always been obtained by pricking the thumb according to the well-known technique of A. E. Wright. By this method, which has been minutely described by Fildes and McIntosh, it is possible to obtain nearly $2 \mathrm{c.cm}$. of blood. The serum is separated from the clot by the centrifuge, and is inactivated by heating at $56^{\circ}$ for 30 minutes. This inactivation has always been carried out within 24 hours after the withdrawal of the blood. Fresh, i.e. active, serum has never been employed. The maximum quantity of serum used has been $0 \cdot 1 \mathrm{c.cm}$.

(2) Haemolytic amboceptor. This was at first a sheep-rabbit amboceptor, but in the latter half of the work, an ox-rabbit haemolysin was used. The latter is preferable and apparently gives rather sharper end-results in some cases. It avoids any source of error which may be due to the natural amboceptor for sheep blood which some specimens of human serum contain in excess. On the other hand, the use of an ox-rabbit haemolysin and ox-corpuscles occasionally gives rise to a difficulty in the technique due to the marked lytic action which some examples of guinea-pig serum possess for ox corpuscles. On 
this account, a control must always be put up when carrying out the titration, and if the guinea-pig serum alone shows any lytic action, it ought to be discarded and another complement used. The amboceptor was invariably titrated each time before use and a quantity equal to $2 \frac{1}{2}$ times the minimal haemolytic dose employed for each tube.

(3) Blood. The blood was collected at the slaughter-house and defibrinated by shaking. A $5 \%$ suspension of the washed blood in salt solution was employed.

(4) Complement. The complement used was the serum of the guinea-pig, obtained by collecting the blood on the previous evening and allowing clotting to take place. The containing-vessel was then placed overnight in the ice-chest, and the serum pipetted off immediately before use. By collecting the blood in this way a larger amount of serum is obtained than by defibrinating the blood and collecting the serum by centrifuging, and also the serum is much freer from haemoglobin staining. There is apparently no diminution in the complementing activity of the serum due to the keeping of the blood overnight. The complement must invariably be titrated before use and its titre noted (see later under "Antigen").

(5) Antigen. This is the most important factor in the test and on its suitability the whole value of the reaction depends. The antigen which has been used exclusively in this work, and the one which has given better results than any of those with which it has been compared, has been a fresh emulsion of living tubercle bacilli in salt solution. It is made up in the following manner:

A young vigorously growing culture of tubercle bacilli on a glycerineegg medium is scraped off the surface of the medium, care being taken not to remove any of the medium along with the culture, and carefully emulsified in $0.85 \%$ salt solution by grinding in a ball and socket mortar, and made up to a strength of $1 \cdot 500$, the bacilli being weighed moist. This emulsion is made up immediately before use and is not sterilised in any way either by heat or by the addition of chemicals. It is important that this emulsion be made freshly each time as its keeping qualities are doubtful, and in comparative experiments carried out with a fresh and an old emulsion on the same sera, at the same time, with the same complement, better and sharper results have been obtained with the fresh emulsion. This point is dealt with more fully in the second part of this paper.

The antigen made in this way must be carefully titrated before 
use. The customary method of carrying out such a titration is to take a fixed amount of complement, generally two minimal lytic doses and to titrate diminishing amounts of the antigen with this, as shown in the following table:

\begin{tabular}{|c|c|c|c|c|}
\hline Antigen & Complement & Salt sol. & Sensitized Blood & salt Sol. \\
\hline 0.5 & 2 M.L.D. & Sufficient to make & 0.5 & To make a total \\
\hline 0.45 & , & $1.5 \mathrm{c.cm}$. in each & ," & bulk of 2.5 c.cm. \\
\hline $0 \cdot 4$ & , & tube & , & in each tube. \\
\hline $0 \cdot 35$ & '. & & ", & \\
\hline e. & , & & ," & \\
\hline
\end{tabular}

The double vertical lines indicate times in the incubator (see later).

This method of titration gives, as a rule, excellent results and allows a suitable dose of antigen, say, half the titre, to be selected for the serum examinations. On occasions, however, the "Eigenhemmung" is so marked that one is forced to use only a very minute amount of antigen in the principal experiment, in order to avoid the unspecific absorption of complement and consequent "summation" effects, and these minute doses of antigen are not sufficient to bring about specific fixation in the presence of very small amounts of antibody.

This phenomenon (of "Eigenhemmung") which may occur even when the same culture is used, which on a previous occasion did not give rise to any "Eigenhemmung," was for a long time a source of difficulty, and it was only when the same antigen was titrated with several complements that it was recognised that the cause was to be sought for, not in the antigen, but in the guinea-pig serum. This difference in guinea-pig sera is very important and is not shown at all in the simple titration of the complement. A serum may be very potent in its complementing power and yet have such a capacity for being absorbed by an emulsion of tubercle bacilli, that it is almost useless in complement fixation work in tuberculosis.

This absorption of the complement is usually ascribed to some property in the antigen and is known as "Eigenhemmung." In order to avoid this undesirable "Eigenhemmung," and at the same time to permit the use of a large dose of antigen, it is necessary to carry out the titration of the complement, both alone, and in the presence of that amount of antigen which will be used in the serum examinations. When this is done, the total amount of complement absorbed by the fixed dose of antigen is at once shown, and it is not necessary to titrate the antigen separately. This is the method which has been employed throughout this work, and the titration has been carried out in one of two ways, viz. 
A. Titration of the Complement in the presence of the Antigen.

\begin{tabular}{l|c|c|c|c|c} 
Complement & Antigen & Salt Sol. & Amboceptor & Blood & Salt Sol. \\
0.05 & 0.5 & To make & $2 \frac{1}{2}$ M.L.D. & 0.5 & To make \\
0.045 &, & 1.5 c.cm. &, &, & 2.5 c.cm. \\
0.04 & $\#$ & in &, &, & in \\
0.035 & $\#$ & each tube &, &, & each tube \\
etc. & & & & &
\end{tabular}

The smallest amount of complement which brings about complete lysis is read as the titre.

The suitable dose of complement for the serum examinations is arrived at by taking the sum of the titre of the complement alone and of the titre of the complement in the presence of the antigen. In this way we know that there will be two lytic doses of complement left in each tube after the unspecific absorption of the complement is complete.

\section{B. Titration of the Complement in the presence of Antigen and} Normal Serum.

\begin{tabular}{|c|c|c|c|c|c|c|}
\hline Complement & Antigen & Normal Serum & Salt Sol. & Amboceptor & Blood & Salt Sol. \\
\hline 0.05 & 0.5 & 0.1 & To make & 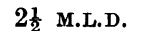 & 0.5 & To make \\
\hline 0.045 & , & , & $1.5 \mathrm{c.cm}$ & , & ," & 2.5 c.cm. \\
\hline 0.04 & , & $"$ & in & ", & ," & in \\
\hline $\begin{array}{l}0.035 \\
\text { etc. }\end{array}$ & , & , & each tube & , & , & each tube \\
\hline
\end{tabular}

The normal serum used in the titration must be inactivated by heating at $56^{\circ}$ for 30 minutes.

Method B is probably the better of the two, and it allows the correct dose of complement to be read at once. It is essential, however, that a "pool" of normal sera should be used in the titration rather than a single serum. This is necessary owing to the differences in normal sera, some increasing the amount of "Eigenhemmung," others definitely diminishing it.

Time. The time allowed for fixation is important, and in this work I have found that 2 or $2 \frac{1}{2}$ hours give much better results than the usual one hour. The results seem to improve from 1 to 3 hours, but after this time there may be some falling off in the activity of the complement. Probably the best results are given when $2 \frac{1}{2}$ hours in the air incubator at $37^{\circ}$ are allowed. It is, of course, essential that similar times and temperatures should be employed in the serum examination as in the titrations.

Temperature. Opinions differ somewhat as to the most suitable temperature for the occurrence of fixation in all complement-fixation 
work. Some observers contend that the reaction takes place best at a temperature of $37^{\circ}$ in a water-bath, whilst other workers maintain that the temperature of the ice-chest is to be preferred. Fildes and McIntosh in their work on the Wassermann reaction have, however, found that the air-incubator is, if anything, superior to both of the above methods and it is their procedure which has been employed throughout this work. After the fixation has occurred, the sensitized blood is added and the tubes returned to the incubator for one hour. At the end of this time the tubes are placed in the ice-chest and the final results read the following morning. All the usual controls must be included in every series of cases.

\section{Results.}

In reading the results obtained by this method in cases of pulmonary tuberculosis, no attention has been paid to small amounts of undissolved cells. Only those cases which gave complete or almost complete fixation have been considered positive. (By "almost complete fixation" is meant that there is only a slight tinting of the fluid whilst the great mass of the red cells remains undissolved.) By adopting this standard it is probable that a number of positive cases have been missed, but this has not been considered very important until the value of the reaction has been more thoroughly worked out.

I. Results in cases of pulmonary tuberculosis with tubercle bacilli in the sputum.

\begin{tabular}{|c|c|c|}
\hline No. of cases & Complement fixation positive & Complement fixation negative \\
\hline Stage I. 109 & . $98(=89 \cdot 9 \%)$ & 11 \\
\hline ., II. 318 & $291(=91.5 \%)$ & 27 \\
\hline , III. 141 & $117(=82.9 \%)$ & 24 \\
\hline Totals 568 & $506(=89.08 \%)$ & 62 \\
\hline
\end{tabular}

There is, apparently, a falling off in the percentage of positive results in the advanced cases (Stage III), but the numbers are too small to draw any conclusions on this point.

\section{Results in apparently healthy persons.}

The serum derived from 45 apparently normal individuals was examined on 204 occasions with invariably negative results. 
III. Results in cases suspected of pulmonary tuberculosis but without tubercle bacilli in the sputum.

(a) Diagnosis of tuberculosis confirmed by the opsonic index or by subcutaneous injections of tuberculin, or by both.

\begin{tabular}{ccc} 
No. of cases & Complement fixation reaction \\
20 & Positive & Negative \\
\hline & 15 & 5
\end{tabular}

(b) Diagnosis of tuberculosis negatived by the opsonic index or by subcutaneous injections of tuberculin, or by both.

$\begin{array}{ccc}\text { No. of cases } & \text { Complement fixation reaction } \\ 63 & \text { Positive } & \text { Negative } \\ 1^{*} & 62\end{array}$

* This case gave only incomplete fixation at first. Three days after the last injection of tuberculin (for diagnosis) the fixation result was completely negative. whilst 14 days later it had again become positive.

\section{Results in diseasés other than pulmonary tuberculosis.}

Only a few cases have been examined, but all have given a negative result unless some other form of tuberculous disease was present. In many of the cases the diagnosis has been confirmed post-mortem.

Cases
Sarcoma of the lung
Carcinoma of the lung
Carcinoma of the stomach
Malta fever
Carcinoma of larynx
Infective endocarditis (two cases)
Carcinoma of liver
Stone in kidney (two cases)
Cystitis
Lupus of pharynx
Tuberculous peritonitis

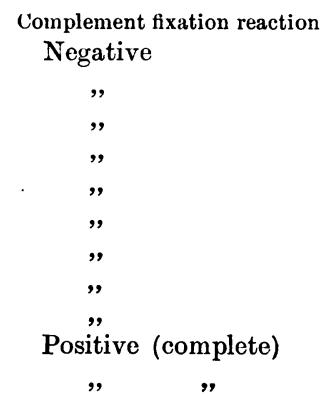

PAR'T II.

This part of the work is supplementary to that already described, and deals with some points in connection with the antigen, and the effects produced by variations in its mode of preparation.

I. Comparative values of different strains of tubercle bacilli as antigens.

In estimating the values of the different strains it is necessary that the comparative experiments should be carried out at the same 
time with the same complement, and that the emulsions should be of the same density.

In this work, eight strains of tubercle bacilli isolated directly from sputum (T. humanus) have been subjected to numerous comparative experiments, and I have not been able to discover any marked differences in the values of the different strains, provided that subcultures of approximately the same age and degree of moisture be employed.

On the other hand, the results obtained with avian bacilli have been much inferior, when compared with the human strains under similar conditions. One experiment, in which a number of tuberculous sera was examined with a fresh human and a fresh avian emulsion, is given in tabular form. The definite superiority of the human emulsion is clearly shown.

$\begin{array}{cccc} & & \text { Human bacilli antigen } & \text { Avian antigen } \\ \text { Case } & 1 & ++++ & ++ \\ \Rightarrow & 2 & ++++ & 0 \\ \Rightarrow & 3 & ++++ & +++ \\ \Rightarrow & 4 & +++ & 0 \\ \Rightarrow & 5 & +++ & ++ \\ " & 6 & ++++ & +++ \\ \Rightarrow & 7 & ++ & 0 \\ (++++=\text { complete fixation; } & 0=\text { complete lysis. })\end{array}$

II. Comparative values of different media in the growth of tubercle bacilli for antigens.

In this investigation four varieties of media were employed:

(1) Glycerine-egg solidified at $80^{\circ}$;

(2) Glycerine-egg coagulated at $100^{\circ}$;

(3) Serum;

(4) Glycerine-potato.

Numerous experiments were carried out under identical conditions, but without being able to establish the definite superiority of any one of the media. On the whole the best results were given when the glycerine-egg medium solidified at $80^{\circ}$ was employed. This was probably due to the fact that this medium allowed of a rapid growth which was readily emulsifiable, whereas the other media gave a slower rate of growth and the culture was more difficult to work up into an even emulsion. 


\section{Comparative values of young and old subcultures as antigens.}

In the experiments designed to investigate this point one strain of bacilli has been employed throughout, grown always on the same medium, viz., glycerine-egg. This strain was isolated directly from sputum by the antiformin method, and after very numerous subcultures could be depended upon to give a fairly abundant growth on the egg medium, in from five days to a week. Numerous sera were examined with antigens made up from cultures of this strain of varying ages, but no marked differences were noticed except when very old cultures (over 12 weeks) were employed. The older cultures seemed to be slightly less valuable, but the differences were so slight that they were probably due to the greater difficulty experienced in preparing an even emulsion from the dry wrinkled growth of the older cultures.

The greater value of the young culture is best seen in the examination of weakly binding sera, very strongly positive sera giving good results with either antigen as seen in the next table:

\begin{tabular}{|c|c|c|c|}
\hline & Serum & $\begin{array}{l}\text { Antigen from culture } \\
78 \text { days old }\end{array}$ & $\begin{array}{l}\text { Antigen from culture } \\
8 \text { days old }\end{array}$ \\
\hline \multirow[t]{5}{*}{ I. } & $0 \cdot 1$ & ++ & $++t+$ \\
\hline & $0 \cdot 05$ & + & $++t$ \\
\hline & $0 \cdot 025$ & o & o \\
\hline & $0 \cdot 0125$ & o & 0 \\
\hline & 0.006 & o & 0 \\
\hline \multirow[t]{6}{*}{ II. } & $0 \cdot 1$ & +++ & ++++ \\
\hline & 0.05 & ++++ & $+++t$ \\
\hline & $0 \cdot 025$ & $+t+t$ & $+t+$ \\
\hline & 0.0125 & +++ & $++t$ \\
\hline & $0 \cdot 006$ & + & +++ \\
\hline & & $(++++=\mathrm{comp}$ & tion.) \\
\hline
\end{tabular}

\section{Effect of age on the antigen.}

The question of the keeping.qualities of these emulsions of tubercle bacilli is an important one owing to the difficulty of having a young vigorous subculture of tubercle bacilli always available, whereas the manipulations would be much simplified if a large quantity of an emulsion could be prepared and stored.

The experiments on this point have been carried out with one strain of tubercle bacilli, and the emulsions have always been stored in the ice-chest. Very little difficulty has been met with in keeping these emulsions sterile, but occasionally they have become contaminated with moulds. All such contaminated antigens have been discarded. 
In considering the effects of age, it is best to divide the description of the results obtained into two parts.

(1) Times less than one week.

In these experiments the antigens were made up not only from the same strain, but also from the same culture tube. For this purpose a very large tube or Roux bottle of the egg medium was used and growth was allowed to continue until the surface of the medium was covered with a dense luxurious layer of growth. The necessary amount of culture was removed by a loop when required, and the antigen prepared and stored in the ice-chest. The culture tube, in the intervals, was sealed and returned to the incubator.

A number of sera were examined with these stored antigens but only very slight differences were noticed, when the length of storage was less than one week. Two of these experiments are shown in the next table:

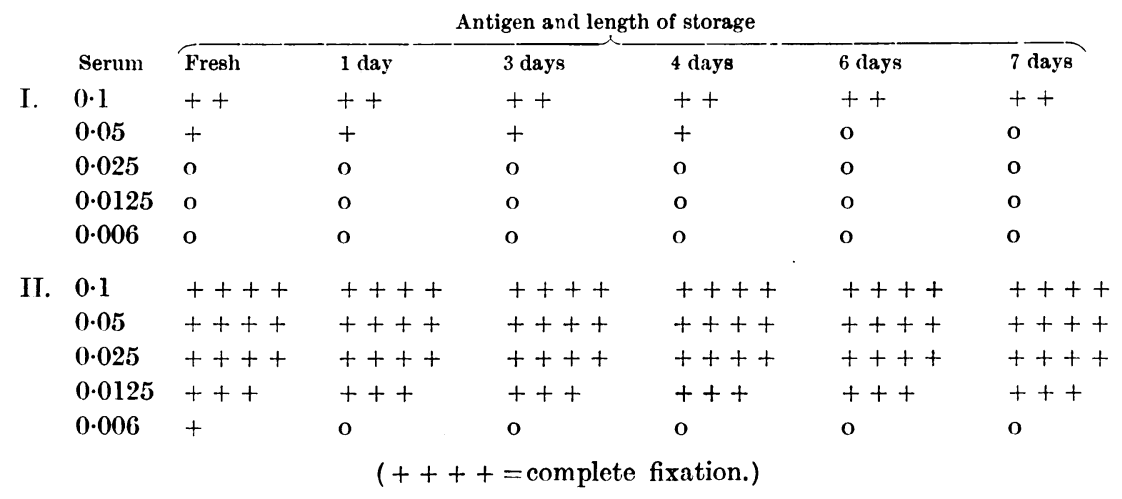

(2) Times longer than one week.

In these experiments several strains of bacilli were employed for comparison, and the antigens were always stored in the ice-chest.

In comparing the values of these stored antigens with a freshly made one, both the serum of patients suffering from pulmonary tuberculosis and the Tuberkulose-Serum, Hoechst, have been employed. Quantitative examinations have been carried out in many of these cases.

Almost invariably better results have been obtained when the freshly prepared antigen was used, as will be seen from the following tables: 
A. Serum from cases of pulmonary tuberculosis.

$\begin{array}{ccc}\text { Case } & \text { With fresh antigen } & \text { With antigen stored for } 20 \text { days } \\ \mathbf{1} & ++++ & 0 \\ \mathbf{2} & +++ & 0 \\ \mathbf{3} & ++++ & + \\ \mathbf{4} & ++++ & + \\ 5 & ++++ & ++ \\ \text { Case } & \text { With fresh antigen } & \text { With antigen stored for } 28 \text { days } \\ 6 & ++++ & ++ \\ 7 & ++++ & +++ \\ 8 & ++++ & +++ \\ 9 & +++ & +++ \\ 10 & ++++ & ++++ \\ 11 & ++++ & \end{array}$

Case 12. Examined quantitatively.

$\begin{array}{lcc}\text { Serum } & \text { Fresh antigen } & \text { Antigen stored for } 28 \text { days } \\ 0 \cdot 1 & ++++ & ++++ \\ 0.05 & ++++ & ++++ \\ 0.025 & ++++ & +++ \\ 0.0125 & ++ & + \\ 0.006 & 0 & 0\end{array}$

B. With the Tuberkulose-Serum, Hoechst.

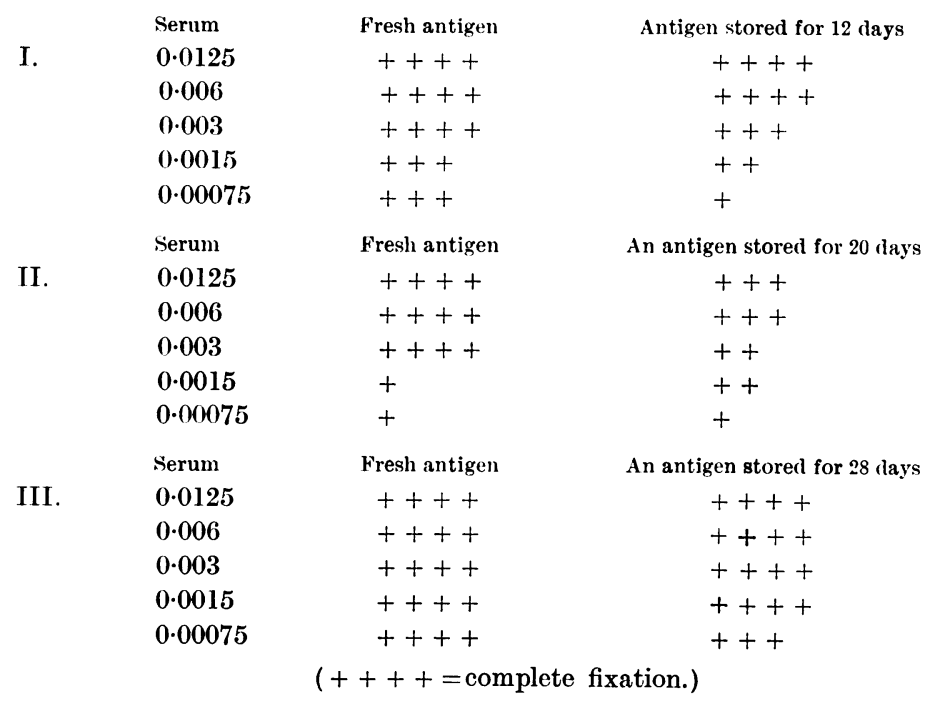

The above tables show that much more constant results are obtained when a freshly made emulsion is used as antigen. The effects of storage for periods less than one week are as a rule not very marked, 
but if longer periods are allowed the results become irregular and errors are quite likely to arise, especially in the case of weakly binding sera. The Tuberkulose-Serum, Hoechst, does not show this superiority of the fresh antigen so well, but this is probably due to the high potency of the serum, which can bring about fixation of complement in the presence of almost any tubercle antigen.

The above tables show that it is necessary, when using this antigen, to make up the emulsion immediately before use, and that it is advisable to use a young subculture of the tubercle bacillus rather than an old dry one.

V. Effect of storage on the amount of "Eigenhemmung" possessed by an emulsion of tubercle bacilli.

This question was investigated by titrating the antigen at different ages with a fixed amount of complement. Two multiples of the minimal lytic doses of each complement being employed and the antigen. being kept in the ice-chest in the intervals. This method, however, gave very unreliable results, the amount of "Eigenhemmung" seeming to fluctuate in either direction irregularly, as will be seen by one of these experiments shown in the next table.

The explanation of this was at first difficult, but the irregularity is, I think, caused by differences in the complements employed, rather than by any changes in the anti-complementary power of the antigen as the result of age:

\begin{tabular}{|c|c|c|c|c|}
\hline \multirow[b]{2}{*}{ Amounts of antigen } & \multicolumn{4}{|c|}{ Ages of the antigen } \\
\hline & I day & 5 datys & 24 days & 31 day \\
\hline $0 \cdot 4$ & ++ & o & ++ & + \\
\hline $0 \cdot 45$ & + & o & + & + \\
\hline $0 \cdot 4$ & + & 0 & + & $o$ \\
\hline $0 \cdot 35$ & o & 0 & + & o \\
\hline $0 \cdot 3$ & o & o & + & 0 \\
\hline $0 \cdot 25$ & 0 & o & o & 0 \\
\hline $0 \cdot 2$ & o & o & o & 0 \\
\hline
\end{tabular}

PART III.

In this section, the effects of exercise and of tuberculin on the antibody content of the serum are described.

I. Effects of exercise on the antibody content of the serum.

This investigation was undertaken to discover whether any fluctuations in the amount of complement-fixing bodies took place as the 
result of exercise, analogous to the fluctuations in the opsonic index. The results were, however, quite negative, the titre of the serum remaining unaffected in either direction, as is seen from the two examples given in the tables.

CASE 1. Pulmonary tuberculosis. T.B. + .

\begin{tabular}{|c|c|c|c|c|}
\hline $\begin{array}{l}\text { Serum } \\
0.1 \\
0.05\end{array}$ & $\begin{array}{l}\text { Before exercise } \\
++++ \\
+++\end{array}$ & $\begin{array}{c}1 \text { hour after } \\
++++ \\
+++\end{array}$ & $\begin{array}{c}6 \text { hours after } \\
++++ \\
+++\end{array}$ & $\begin{array}{c}24 \text { hours after } \\
++++ \\
+++\end{array}$ \\
\hline & sE 2. $P u$ & ary tuber & T.B. & \\
\hline Serum & Before exercise & 1 hour after & 6 hours after & 24 hours after \\
\hline $0 \cdot 1$ & o & o & o & o \\
\hline $0 \cdot 05$ & o & o & o & o \\
\hline
\end{tabular}

\section{Effect of tuberculin.}

A number of experiments have been carried out to investigate this question, partly in normal, partly in tuberculous subjects.

In the majority of these cases the tuberculin had been given subcutaneously for diagnosis, but in a few cases of definite tuberculosis the reaction was carried out before and also at the end of a course of tuberculin treatment.

The results obtained have been very irregular, especially in those persons who received cumulative doses of old tuberculin for diagnosis. In all these cases the test was completely negative before the administration of the tuberculin, whereas afterwards some remained negative, others became positive temporarily. I have no explanation to offer for this change in the reaction, as most of these patients had no other evidence of tuberculosis.

A few of the results obtained are shown in the table:

$\begin{array}{ccc}\text { Case } & \text { Result before tuberculin } & \text { Result after tuberculin } \\ 1 & 0 & 0 \\ 2 & 0 & 0 \\ 3 & 0 & 0 \\ 4 & 0 & +++ \\ 5 & + & ++++ \\ 6 & 0 & + \\ 7 & 0 & + \\ 8 & 0 & ++ \\ 9 & 0 & + \\ 10 & 0 & +++ \\ 11 & 0 & 0 \\ 12 & 0 & 0 \\ 13 & 0 & + \\ 14 & 0 & \\ (++++=\text { complete fixation of complement.) } \\ (0=\text { complete lysis, } i . e ., \text { no fixation of complement.) }\end{array}$

Journ. of Hyg. xv 
In the cases of definite pulmonary tuberculosis with tubercle bacilli in the sputum, the usual result of a long course of tuberculin treatment was to cause an increase in the amount of complementfixing bodies in the serum, provided that these bodies had been present in fair amount before the treatment was commenced. On the other hand, in those cases which had given a negative result in spite of their tubercle bacilli, the tuberculin treatment did not seem to cause any development of these bodies in the few cases investigated.

\section{SUMMARY.}

1. A fresh emulsion of tubercle bacilli in salt solution when used as the antigen will give about $90 \%$ of positive results in cases of pulmonary tuberculosis, whilst in healthy persons the result is negative.

2. In cases suspected of pulmonary tuberculosis, the results of this test conform closely to those given by a combination of the opsonic index test with injections of tuberculin, and so may be of value in diagnosis.

3. Diseases other than tuberculosis have always been negative, but only a small number of such cases have been examined.

4. An emulsion of tubercle bacilli when used as an antigen must be freshly prepared immediately before use, and should be made from a young subculture.

5. Owing to the differences in complements, they should always be titrated in the presence of the antigen, and probably also in the presence of both antigen and normal serum.

6. Exercise does not seem to affect the result of the reaction in any way, but a course of tuberculin treatment leads to an increase in the complement-fixing bodies of the serum in some cases.

\section{REFERENCES.}

1. Bondet et Gengou (1903). Compt. rend. de l'Académie des Sciences, cxxxvir. 351.

2. Wassermann und Bruck (1906). Deutsche med. Wochenschr. xxxir. 449.

3. Citron (1907). Berlin. klin. Wochensch. III. 1135.

4. Morgenroth und Rabinowitsch (1907). Deutsche med. Wochenschr. xxxill. 750.

5. Hammer (1912). Münch. med. Wochenschr. LIX. 1750.

6. Bang und Andersen (1913). Centralblatt f. Bakt. Originale lxix. 517.

7. Besredka et Manoukhine (1914). Compt. rend. de la Soc. de Biolog. Lxxvi. 180.

8. Calmette et Massol (1914). Annales de l'Institut Pasteur, xxviII. 338.

9. Renaux (1914). Compt. rendu de la Soc. de Biolog. Lxxvi. 864.

10. Fildes and McIntosh (1913). Brain, xxxvi. p. 193. 